\title{
Recognition memory for literal, figurative, and anomalous sentences
}

\author{
ROBERT G. MALGADY \\ State University of New York, Brockport, New York 14420 \\ and \\ MICHAEL G. JOHNSON \\ University of Tennessee, Knoxville, Tennessee 37916
}

\begin{abstract}
In a yes-no recognition memory experiment, subjects studied literal, figurative, and anomalous sentences and were later required to identify target items among a comparable set of distractors. For target items, recognition probability was higher for literal sentences than for anomalous sentences, and lowest for metaphors. The false alarm rate was highest for metaphors, next highest for literal sentences, and lowest for anomalous sentences. Recognition performance was negatively correlated with the "figurativeness" of sentences. Results were interpreted in support of a feature transfer operation in processing meaning.
\end{abstract}

More than a decade ago, Dwight Bolinger (1965) argued that an adequate theory of language comprehension must reconcile the problem of how a language user apprehends figurative meaning in metaphorical speech, which often arises in everyday discourse. Since then linguists have attempted to develop formal criteria for distinguishing literal, figurative, and anomalous sentences (e.g., Bickerton, 1969; Weinreich, 1966), but virtually all psychological inquiry into the cognitive processes underlying language comprehension and memory has focused on the study of literal and anomalous sentences without considering metaphorical sentences.

The term "metaphor" generally refers to the description of one object or event in terms of some literally disparate concept, where the comparison is justified by some underlying similarity. In other words, metaphor exercises one's perception of the similarity of apparently different words. It is conventional, at least in literary circles, to distinguish between the "tenor" or subject of a metaphor (e.g., The dew is a veil on my head) and the "vehicle" or predicate (e.g., veil) which carries the tenor. One theory of metaphor comprehension suggests that the semantic features which encode the meaning of the vehicle are transferred to the tenor. Despite the fact that some features are mutually contradictory, features shared by both terms are increased in salience, while contradictory features are obscured (Smith, Rips, \& Shoben, 1974; Johnson, Malgady, \& Anderson, Note 1). According to this view, overlapping semantic features create a basis for the figurative meaning of the tenor, and contradictions between the literal meaning of the tenor and vehicle are de-emphasized. Empirical support for this characterization of figurative language was evident in several recent studies dealing with comprehension and apprecia- tion of metaphor (Malgady, 1976; Malgady \& Johnson, 1976; Johnson et al., Note 1).

As regards memory for figurative language, Verbrugge (in press) has suggested that a linguistic abstraction of the resemblance between the tenor and vehicle (the "ground") is what is stored in memory. Verbrugge demonstrated that symbolic analogy prompts which contained the same figurative relationship between tenor and vehicle as target analogies facilitated recall in comparison to a free recall situation, in spite of the fact that there were no other similarities between prompts and target items. In a receognition memory study, Harris (Note 2) reported that Shakespearean metaphors were recognized more often than literal paraphrases of the metaphors and also that the false alarm rate was higher for metaphors. These findings were interpreted in terms of the increased retrieval possibilities invoked by the "interacting semantic domains" of metaphor.

Although previous studies provide information about comprehension of and memory for figurative language, they provide little direct evidence for a comparison of factors involved in processing literal, figurative, and anomalous sentences. The purpose of the present study was to investigate recognition memory for literal sentences, metaphors, and anomalous sentences. Specific questions addressed in this study were: (1) how well are metaphors remembered relative to literal and anomalous sentences, (2) does confidence in recognition performance differ as a function of sentence type, and (3) what is the relationship between the figurativeness of sentences and recognition performance.

\section{METHOD}

Subjects

Subjects in the study were 33 undergraduates enrolled in an 
introductory psychology course. All subjects participated in the experiment on a voluntary basis.

\section{Materials}

Simple active declarative sentences were formed according to the sentence frame: Noun-is/are-(a)-noun. For each of three subject nouns, a set of 40 predicate nouns were selected which represented literal, figurative, and anomalous relationships with a given subject noun. Noun pairs judged as literally related (e.g., blossoms-flowers) were synonyms drawn from several standard English dictionaries. Figuratively related noun pairs (e.g., dew-veil) were collected in a previous study of figurative language by Johnson et al. (Note 1). Anomalous noun pairs (e.g., robes-trucks) were generated by randomly pairing seemingly unrelated nouns. All nouns were of relatively high frequency and were familiar to college-age subjects.

Twenty-one subjects classified each of the 120 sentences as either a literal statement, a figure of speech, or as nonsense. Subjects classified the sentences according to their own criteria, based upon their sense of what the terms literal, figurative, and nonsense mean. Based upon the modal classification of each sentence, all sentences were categorized as either literal, figurative, or anomalous and 30 sentences were randomly selected from those in each category which were reliably scaled in terms of a chi-square criterion. Half were used as target items on an acquisition list and half were used as distractors in the recognition phase.

\section{Procedure}

The 45 target sentences on the acquisition list were typed on $3 \times 5$ in. cards and presented to subjects in decks. In order to minimize primacy and recency effects, three filler sentences (one of each sentence type) preceded and followed the presentation of target sentences in the acquisition phase. Each subject studied the sentences in a different random order.

In the acquisition phase, 12 subjects were told to study each sentence and that later they would be asked some questions about the sentences. Subjects studied each sentence for $5 \mathrm{sec}$ and proceeded through the decks in response to an auditory signal given by the experimenter. A 2-min "rest period" separated the acquisition and recognition phases of the study.

In the recognition phase, subjects were given booklets containing all 90 sentences (45 target items and 45 distractors). Booklets consisted of 10 pages of sentences (nine per page), an equal number of different sentence types appearing on each page. The proportion of recognition types (target vs distractor) was varied randomly across pages. For each subject, a different random order of pages was determined.

Using a yes-no recognition procedure, subjects were instructed to read a given sentence and then decide whether it appeared in the deck they had just studied. Subjects also indicated the confidence they had in their judgments by saying either "sure" or "probable" yes or no. Subjects were tested individually and in pairs. No time limit was imposed during the recognition phase.

\section{RESULTS}

Subjects' responses on the recognition test were scored for accuracy and confidence (percentage of "sure" ratings). Multivariate analysis of variance was used to assess the effects of sentence type and recognition type on these two measures. Separate statistical analyses were conducted treating subjects and items as random sampling variables.

The multivariate analyses revealed a significant effect of sentence type across both subjects $[F(4,42)=$

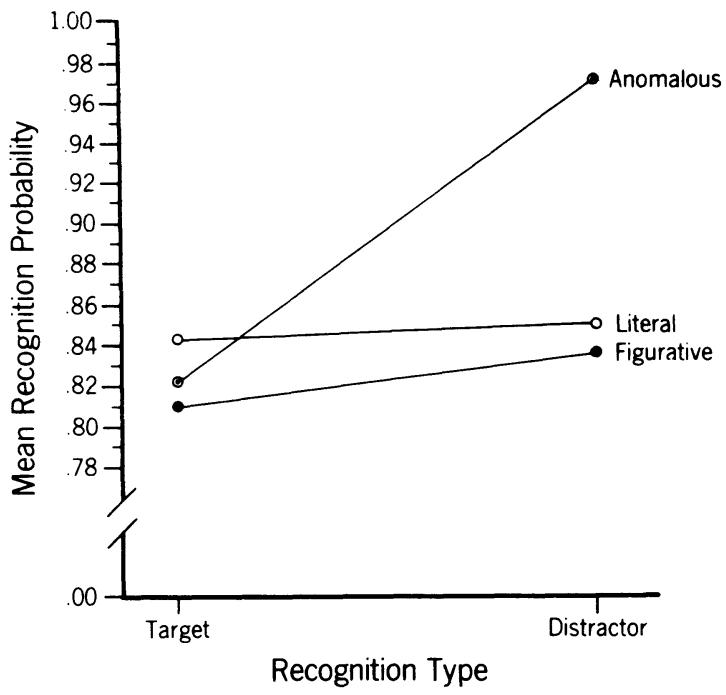

Figure 1. Mean recognition probability (proportion correct) for sentence type as a function of recognition type.

9.65, $\mathrm{p}<.001]$ and items $[\mathrm{F}(4,166)=2.65, \mathrm{p}<.05]$. Recognition types differed significantly in the item analysis $[F(2,166)=6.49, \mathrm{p}<.001]$ but not in the subjects analysis $[F(2,20)=3.25, p>.05]$. The Sentence Type by Recognition Type interaction was also significant, for subjects $[F(4,42)=2.98, p<.05]$ and for items $[F(4,166)=3.77, p<.01]$. Further analysis indicated that, for target items, sentence type accounted for only $17 \%$ of the predicable variance in the subjects analysis and $10 \%$ of the predictable variance in the items analysis. Conversely, for distractors, sentence type accounted for $83 \%$ and $90 \%$ of the predictable variance in respective analyses.

Mean recognition probability is presented in Figure 1 for sentence type as a function of recognition type. Figure 1 shows that, on the average, distractor sentences were recognized more easily than target items. Literal targets were recognized better than anomalous sentences; however, recognition probability was lowest for figurative targets. For distractors, anomalous sentences were recognized better than literal sentences, while the false alarm rate was highest for metaphors.

Mean confidence ratings for correct responses are shown in Figure 2 for sentence type as a function of recognition type. For target items, subjects had less confidence in their correct recognition of literal sentences than either anomalous or figurative sentences, while the latter were not different. For distractors, the pattern of results was the same as for recognition probability. Subjects had most confidence in correctly rejecting anomalous sentences, next most confidence in literal sentences, and they were least sure of their rejection of figurative sentences.

Data from the sentence classification task described earlier were used to scale the 90 sentences with respect to their degree of "figurativeness." The figurativeness 


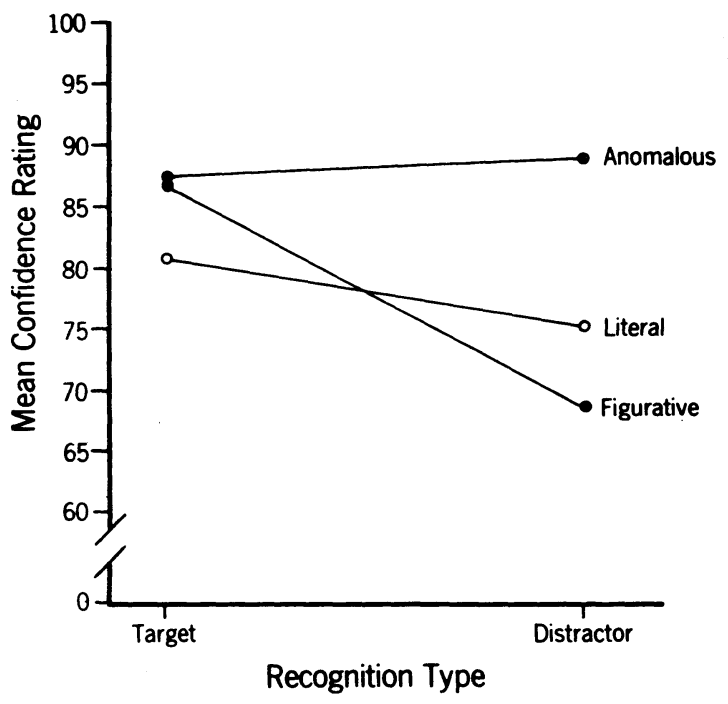

Figure 2. Mean confidence rating (percentage of "sure" responses) for correct recognition of sentence types as a function of recognition type.

of a sentence was calculated as the percentage of subjects $(N=21)$ classifying the sentence in the "figure of speech" category. Recognition scores were recalculated for all sentences weighting recognition probability by confidence rating. A regression analysis conducted across items indicated that recognition scores for distractors were negatively correlated with figurativeness scores $(\mathrm{r}=-.545, \mathrm{p}<.001)$. Although this correlation was also negative for target items, it was only marginally significant $(\mathrm{r}=-.279, \mathrm{p}<.07)$. Thus, recognition performance decreased linearly with the figurativeness of sentences.

\section{DISCUSSION}

Results of the present study are in agreement with a portion of Harris' (Note 2) investigation of recognition memory for Shakespearean metaphors and literal paraphrases of metaphors. The results for distractor sentences replicate the earlier findings. of Harris, who also found a higher false alarm rate for metaphors relative to nonmetaphors. One explanation offered by Harris is that subjects might be biased toward choosing metaphors because they are more difficult to interpret than literal sentences. This speculation seems unlikely, however, since in the present study such a response bias should have led to the highest false alarm rate for anomalous sentences.

An alternative explanation suggested by Harris (Note 2) is more consistent with the present findings and the model of metaphor understanding proposed by Smith et al. (1974) and Weinreich (1966). The tendency to falsely recognize metaphors may reflect the fact that metaphors involve an "additional semantic domain" and, therefore, more semantic information to falsely associate with. In other words, the transfer of semantic features from vehicle to tenor may result in a larger feature representation of the tenor in the case of a metaphor compared to the feature representation of a literal sentence. For this reason, the probability of confusion between distractors and meanings of the tenor encoded during acquisition is greater for metaphors. On the other hand, the nouns making up anomalous sentences are so dissimilar that they share few, if any, semantic features; hence, their associated feature sets are almost entirely contradictory. The two "semantic domains" compared in anomalous sentences are not combined into a meaningful representation of the subject of the sentence; that is, meaning cannot be construed. Unlike the meaning of literal or figurative sentences, which have a single feature representation in memory, anomalous sentences may not be integrated into a unitary feature representation. The observation of fewest false recognitions for anomalous sentences might therfore reflect the lower probability of confusing two distinct meanings (two separate nouns) than confusing one integrated meaning with other meanings encoded during acquisition.

Contrary to the present findings, Harris (Note 2) found that figurative targets were correctly recognized more of ten than literal targets. In the present study, literal sentences were recognized more accurately than anomalous sentences, but rather surprisingly, figurative sentences were remembered least of ten. An explanation of higher recognition probability for literal sentences compared to anomalous sentences might appeal to the greater meaningfulness, familiarity, or perhaps higher internoun similarity of the former sentence type. Clearly, however, figurative sentences are more meaningful, less unusual, and have higher tenor-vehicle similarity than anomalous sentences. These findings may be attributed to more complex information processing activated by the intermediate level of similarity between nouns compared in metaphors compared to the high degree of similarity in literal sentences and low similarity in anomalous sentences (cf. Smith et al., 1974).

\section{REFERENCE NOTES}

1. Johnson, M. G., Malgady, R. G., \& Anderson, S. Some cognitive aspects of metaphor interpretation. Paper presented at the meeting of the Psychonomic Society, Boston, November 1974.

2. Harris, R. J. Recognition memory for Shakespearean metaphor. (Tech. Rep. 75-3). Manhattan, Kans: Kansas State University, Human Information Processing Institute, 1975.

\section{REFERENCES}

Bickerton, D. Prolegomena to a linguistic theory of metaphor. Foundations of Language, 1969, 5, 34-52.

Bolinger, D. The atomization of mearing. Language, 1965, 41, 555-573.

Malgady, R. G. Category size, feature comparison, and the comprehension of figurative propositions. Perceptual and Motor Skills, 1976, 42, 811-818.

Malgady, R. G., \& Johnson, M. G. Modifiers in metaphors: Effects of constituent phrase similarity on the interpretation of figurative sentences. Journal of Psycholinguistic Research, 1976, 5, 43-52.

Smith, E. E., Rips, L. J., \& Shoben, E. J. Semantic memory and psychological semantics. In G. H. Bower (Ed.), The psychology of learning and motivation (Vol. 8). New York: Academic Press, 1974.

VERBRUGGE, R. R. Resemblances in language and perception. In R. Shaw \& J. Bransford (Eds.), Perceiving, acting, and comprehending: Toward an ecological psychology. Hillsdale, N.J: Lawrence Erlbaum, in press.

Weinreich, U. Explorations in semantic theory. In $T$. Sebeck (Ed.), Current trends in linguistics: Theoretical foundations (Vol. 3). The Hague: Mouton, 1966.

(Received for publication October 18, 1976.) 